\title{
Editorial
}

\section{Doing Educational Research: Culturally, Scientifically, Ethically, and Transdisciplinarily}

The contributors of this issue of the journal represent different geographical localities, like Germany, The United States of America, Australia, Iran, Indonesia, Japan, Turkey, Saudi Arabia, Portugal, Austria, the United Kingdom, Italy, and Finland. They focus of diverse range of issues of sustainable development in the face of ambivalence, chaos and uncertainty. The contributions of this volume of the journal highlight complex issues that require involvement of a multitude of actors and societal subsystems by revealing multidimensional and dynamic concept of sustainability. Regardless the context all of the authors discuss diverse issues of unsustainable development to which there are no simple solutions. Instead of dealing with small, fragmented, isolated and localized issues, we need to focus of the phenomena of unsustainable development and look for the root causes of unsustainability by designing a comprehensive framework for tackling smaller local issues. Problems in the local context need to be tackled, reinterpreted and restructured from the ecosystem perspective. Local challenges require creative and system approaches by including elements of responsibility, public engagement and responsibility. The governance of sustainable changes requires empowerment capacities, communityoriented governance modes, and coevolution of sustainability initiatives where only responsible changes can lead to a sustainable development. By proposing open ended futures in an open-ended way may lead to achieving sustainable objectives in an unsustainable overall context. Local cases offer the examples of sustainable impacts of their projects leading to a coevolutionary and emergent changes that forces social systems for sustainable transformations.

The article by Simo Häyrynen, Tuula Keinonen and Sirpa Kärkkäinen focuses on how local cultures can be seen in educational doctrines and in the practices of science education, and what impact do they have on the development of environmental citizenship. The viewpoint is that a contextual resource doctrine provides a framework of people's environmental values, meaning both capacity to fulfil the requirements of the doctrine and ability to critically reflect it by rational-scientific arguments. Thus, the article applies the idea of 'trans-contextualisation' and uses examples which highlight student's senses of places in relation to environmental issues. The results show that if cultural traditions, anecdotal and local knowledge are not incorporated in the expert scientific knowledge, sustainability transition is constantly in danger for remaining an isolated activity in the shadow of resource realism.

The study by Ediyanto Ediyanto, Norimune Kawai, Masashi Hayashida and Nagako Matsumiya, Md Abu Bakor Siddik, and Areej Talea Almutairi reflects results entitled "Indonesian teachers' attitudes toward inclusive education" that have clearly illustrated that teachers in Indonesia have a moderate interest towards inclusive education. These results were obtained by measuring using an instrument called TATIS. The current study has shown teacher attitudes with three aspects, namely teachers' perceptions of students with mild to moderate disabilities (POS), attitudes toward beliefs about the efficacy of inclusion (BEI) and beliefs about professional roles and responsibilities (PRF). After the 
teacher's attitudes were clearly explained, then their attitudes were compared based on gender, province, type of school, level of school, last education, teaching experience, and teaching experience with special needs students. Finally, in the current study, it is also known that there is a need to improve teacher attitudes towards inclusive education with sustainable training programs.

The paper by Alireza Bonyadi, Mehdi Kheyrollahi and Minoo Bonyadi has focused on code-switching in EFL classroom discourse. The study qualitatively investigated teachers' perceptions on code-switching in their classrooms addressing two research questions, namely what types of code-switching EFL teachers were practicing in EFL classrooms and what were their perceptions on their code-switching. The analysis of the data collected through manual and electronic observations as well as structured interviews, indicated that intra-sentential and inter-sentential types of code-switching were practised throughout the classroom teaching processes. The main motives for resorting to code-switching were found to be EFL students' lack of linguistic proficiency, keeping solidarity with the students and managing the classrooms.

Geert Franzenburg in his article describes how methods of intersubjective dialogue can make partnership sustainable. After evaluating dialogue strategies since biblical times, he analyzes dialogical texts of the last centuries concerning their "transcultural" perspective. He highlights in his research that intersubjective dialogue - as vicarious learning by encounter - combines different spheres (thoughts and emotions, cultures, transcendent and immanent sphere); intersubjective dialogue, therefore, facilitates new ideas (projects) and equal/symmetric participation; it, thus, fosters self-esteem and responsibility of the partners by applying particularly non-violent, creative, democratic, and transcultural models of contact, needs- and awareness oriented (verbal and non-verbal) communication.

The paper by Nanako Sakai focuses on interreligious education based on the Ten Virtuous Deeds of the Bodhisattvas. A Bodhisattva in Sanskrit signifies an enlightened being who seeks Buddhahood through the practice of the perfect virtues (paramita). There are many oppressed women in Asian countries whose voices are not heard in society. By illustrating the reality of oppressed women in Asia, this study explores how Buddhist feminism can foster spiritual growth and morality. Drawing mainly from the works of contemporary women thinkers and artists whose works exemplify these perspectives, this essay considers the broader meaning of sustainability and how the beauty of human nature and spirituality can be cultivated from a Buddhist feminist perspective. Although the Ten Virtuous Deeds of Bodhisattva are the minimum moral precepts, art can be a tool to transform negative energy against oppressed women into empowerment.

The paper by Berise Heasly analyses the connections and overlaps of the whole field of Education in $21^{\text {st }}$ century terms. The concept of Edu-Tensegrity gives the opportunity to integrate our familiar concepts of Sustainability and Securitability within the huge plane of Education as it is practised universally. So many academic disciplines, so many new skills, so much emerging knowledge must be examined and sifted to allow us all to benefit from the diversity and breadth of emerging knowledge. The whole process therefore of understanding where different educational concepts and skills fit in the overall schema is needed. The quality of thinking patterns is central to our ability to analyse, to collaborate, to communicate and to research. So the whole community of experts in all fields of modern and ancient frameworks of scientific fields needs our attention. It is a huge task, and relies on the contribution of all educators, whose levels 
of expertise needs promotion, upgrading and acknowledgement. This is a crucial element of the work done in every research project we complete and advertise. Researchers as a community central to Edu-tensegrity are the glue we use to negotiate problems, pandemics and paradigm changes.

The paper by Takao Arai, Kengo Saito and Yuji Hirai deals with topicality COVID-19 as encountered in strategy meetings. The authors conducted text mining and principal component analysis of the minutes of the local government web conference on COVID-19 countermeasures held in Hachioji City, Tokyo, Japan, to objectify its contents. As a result, we were able to objectively capture the changing reality of meetings in accordance with the infection status in the region as a dynamic change on a new plane. This local government conference is an initiative related to "community health care collaboration" in "Goal 3: Ensure healthy lives and promote well-being for all at all ages", one of the 17 SDGs. The results of this study can contribute to the implementation of Education for Sustainable Development, or ESD, which stands for group work and education by diverse people who will be the creators of the SDGs, by presenting a new method of evaluating the effectiveness of the conference.

In their paper, Emine Kübra Pullu and Mehmet Nuri Gömleksiz present an experimental study that they conducted with associate degree computer technology students with an authentic task-oriented pretest-posttest control group in programming teaching. Authentic tasks consist of activities based on understanding and solving real-life problems. They made authentic task-oriented practices in programming teaching with experimental group students $(n=30)$ for 8 weeks. With the control group students $(n=33)$, the lessons were conducted with traditional practices. Three different scales and an achievement test developed by the researchers were used as pretest and posttest. As a result of the research, they concluded that authentic task-oriented practices in programming teaching positively affect students' academic achievement, attitudes towards programming, problem solving and creative thinking skills.

The paper by Telma André, Dárida Maria Fernandes, Maria Inês Pinho reflects research project developed at PES that was based on the European project UKIDS (Erasmus +). The paper reflects challenges of the project that include a variety of tasks to work on aspects such as initiative, motivation and innovation, as well as trust and responsible social participation. The research project was developed at the Supervised Teaching Practice (PES) and was inspired on the European project UKIDS (Erasmus+). Specifically, in the Trash Value challenge, which proposes an interdisciplinary knowledge and responsible social participation giving new life to waste and respecting a sustainable environment. Based on the implementation of this challenge and using the egg cartons, it was possible to investigate how this material potentiates the development of social skills, reasoning and mathematical communication of children in the $4^{\text {th }}$ year of schooling. The research methodology included different techniques and instruments for data collection and was organized in the form of multimodal narration. At the end was possible to verify that the Trash Value challenge promoted the development of social skills, with a greater focus on cooperation, self-control and responsibility. Consequently the problemsolving and mathematical communication skills improved the children's environmental awareness. The authors conclude that implementation of this type of projects / challenges allows operationalizing the integration and articulation of the curriculum, resulting in more meaningful learning for children. 
The paper "Integrating Sustainability-Oriented Ecologies of Practice across the Learning Cycle: Supporting Transformative Behaviours in Transgenerational, Transnational and Transdisciplinary Spaces" written by the group of international scholars, examines the complexities associated with effectively and comprehensively tackling the climate change crisis. Focusing on the need for education, the authors discuss a model of Education for Sustainable Development that supports the development of competencies, coalition building and the capacity to support and maintain positive action. Drawing upon principles highlighted by the United Nations, the paper outlines the breadth and depth of knowledge required to support transformative ESD. Firstly, enhancing comprehensive knowledge that develops cognitive, affective and axiological dimensions and proficiency. This enhances critical engagement with information and enables individuals to act responsibly and align with others in coalition building. This second element is critical for change to be effective as collaborative partnership has been one of the most challenging barriers preventing positive action on the catastrophe of climate change. Finally, the paper emphasizes the need to develop the competencies for supporting collective action, which will enable sustained action across transnational, transdisciplinary and transnational boundaries.

We would like to express our gratitude to all the authors who offered their contributions to this issue of the journal as well as to all the reviewers and the language editors. In thanking all contributors, we acknowledge the function and role of researchers, who identify educational roadblocks, and shape questions designed to uncover hurdles, identifying options to provide processes which can facilitate problem-solving as well as management of interim moves aimed at the best ethical action possible.

Here we find the intentional focus in the educational secular state, allowing us to secure our best efforts to promote the 'common good', hence the emphasis on the concept of Securitability. In our efforts to prolong that securitability, we move deliberately towards the embedding of such educational improvements, hence the emphasis on the central concept of Sustainability.

It is worth noting that these $21^{\text {st }}$ century research systems, both quantitative and qualitative in nature, veer also towards religious aims for education integrity, especially in the Abrahamic systems. Parallels in ethical integrity, partnered with equality and understanding of diversity, help us as educational leaders to follow with distinction the aims and intentions of the sustainable development goals. And this is increasingly necessary in the face of climate change, highlighted in the Glasgow conference, 2021.

Berise Heasly, Dzintra Iliško 